\title{
Statistical distribution of elapsed times and distances of seismic events: the case of the Southern Spain seismic catalogue
}

\author{
M. D. Martínez ${ }^{1}$, X. Lana ${ }^{2}$, A. M. Posadas ${ }^{3,4}$, and L. Pujades ${ }^{5}$ \\ ${ }^{1}$ Departament de Física Aplicada, Universitat Politècnica de Catalunya, Av. Diagonal 649, E-08028 Barcelona, Spain \\ ${ }^{2}$ Departament de Física i Enginyeria Nuclear, Univ. Politècnica de Catalunya, Av. Diagonal 647, E-08028 Barcelona, Spain \\ ${ }^{3}$ Departamento de Física Aplicada, Universidad de Almería, E-04120, Almería, Spain \\ ${ }^{4}$ Instituto Andaluz de Geofísica y de Prevención de Desastres Sísmicos, Universidad de Granada, E-18080, Granada, Spain \\ ${ }^{5}$ Departament d'Enginyeria del Terreny, Cartogràfica i Geofísica, Universitat Politècnica de Catalunya, Gran Capità s/n, \\ E-08034, Barcelona, Spain
}

Received: 12 July 2004 - Revised: 29 December 2004 - Accepted: 1 February 2005 - Published: 8 February 2005

Part of Special Issue "Seismicity pattern dynamics"

\begin{abstract}
Several empiric cumulative distributions of elapsed times and distances between seismic events occurred in the Southern Iberian Peninsula from 1985 to 2000 (data extracted from the seismic catalogue of the Andalusian Institute of Geophysics) are investigated. Elapsed times and distances between consecutive seismic events of the whole catalogue, taking into account threshold magnitudes of 2.5, 3.0, 3.5 and 4.0, and of five seismic crises, without distinguishing magnitudes, are investigated. Additionally, the series of distances and elapsed times from the main event to every aftershock are also analysed for the five seismic crises. Even though a power law is sometimes a satisfactory model for the cumulative distribution of elapsed times and distances between seismic events, in some cases a fit with a Weibull distribution for elapsed times performs better. It is worth of mention that, in the case of the seismic crises, the fit achieved by the power law is sometimes improved when it is combined with a logarithmic law. The results derived might be a contribution to a better representation of the seismic activity by means of models that could be based on random-walk processes.
\end{abstract}

\section{Introduction}

The analysis of the space-time patterns of the seismic activity is a relatively complex problem. Several researches are devoted to the spatial and temporal clustering of the seismicity (Ouchi and Uekawa, 1986; Smalley et al., 1987 and Chen et al., 1998, among others). The chaotic and dissipative be-

Correspondence to: M. D. Martínez

(dolors.martinez@upc.edu) haviour of the elapsed times and how can be characterised the predictability of the seismicity in terms of Lyapunov exponents have been also analysed for instance by Correig et al. (1997) by using the reconstruction theorem (Takens, 1981). More recently, nonlinear analyses of sequences of magnitude and interevent time intervals have been performed by Matcharashvili et al. (2000) and differences in the temporal distribution of small earthquakes before and after large events have been investigated by Matcharashvili et al. (2002). In the first case, significant evidence of low nonlinear dimension structure is detected. In the second case, it is verified that the low nonlinear dimension structure of the temporal distribution of earthquakes changes remarkably before and after the largest events. It is also worth of mention the book of Goltz (1998), where concepts of fractal and chaos are introduced and fractal and chaotic properties of earthquakes are broadly analysed.

From the point of view of a stochastic process, it has to be mentioned the model of the continuous time random walk, CTRW (Bouchaud and Georges; 1990) and Lévy distributions (Gnedenko and Kolmogorov, 1954; Feller, 1996), which try to establish relationships between interevent waiting times and hypocenter location. An illustrative application can be found in Sotolongo-Costa et al. (2000) and Posadas et al. (2002).

The main objective of the present study is to obtain statistical models to describe the elapsed times and distances between earthquakes occurred in Southern Spain. These results will be compared with those derived from several seismic crises occurred in the same area. Although the distribution of the elapsed times between consecutive events has been extensively studied (Smalley et al., 1987; Sornette and Knopoff, 1997; Wang and Kuo, 1998; Corral, 2003, 2004), 
Table 1. Range of magnitudes, $\Delta m$, recording period, $\Delta t$, number of events, latitude and longitude intervals, parameters $b$ of the GutenbergRichter law and $p$ of the Omori's law, and fractal dimension $D_{S}$ of the spatial earthquake distribution for the five seismic crises of Adra, Agrón, Alborán, Antequera and Loja. Uncertainties on $D_{S}, b$ and $p$ are also specified.

\begin{tabular}{|c|c|c|c|}
\hline & Adra & Agrón & Alborán \\
\hline$\Delta m$ & $5.0-1.5$ & $4.0-1.5$ & $4.5-2.5$ \\
\hline \multirow[t]{2}{*}{$\Delta t$} & 23 Dec. 1993 & 5 Dec. 1988 & 2 July 1997 \\
\hline & 16 March 1994 & 15 Dec. 1998 & 24 Dec. 1997 \\
\hline events & 346 & 101 & 56 \\
\hline latitude & $36.4^{\circ} \mathrm{N}-37.0^{\circ} \mathrm{N}$ & $36.99^{\circ} \mathrm{N}-37.05^{\circ} \mathrm{N}$ & $36.3^{\circ} \mathrm{N}-36.7^{\circ} \mathrm{N}$ \\
\hline longitude & $2.5^{\circ} \mathrm{W}-3.3^{\circ} \mathrm{W}$ & $3.8^{\circ} \mathrm{W}-3.9^{\circ} \mathrm{W}$ & $2.8^{\circ} \mathrm{W}-3.4^{\circ} \mathrm{W}$ \\
\hline$b$ & $0.87 \pm 0.02$ & $0.97 \pm 0.02$ & $0.86 \pm 0.03$ \\
\hline$p$ & $0.983 \pm 0.001$ & $0.89 \pm 0.01$ & $0.86 \pm 0.01$ \\
\hline \multirow[t]{2}{*}{$D_{S}$} & $2.10 \pm 0.03$ & $2.01 \pm 0.04$ & $1.79 \pm 0.01$ \\
\hline & Antequera & Loja & \\
\hline$\Delta m$ & $3.5-2.5$ & $3.5-2.0$ & \\
\hline \multirow[t]{2}{*}{$\Delta t$} & 7 June 1989 & 3 Feb. 1985 & \\
\hline & 9 June 1989 & 13 June 1985 & \\
\hline events & 79 & 146 & \\
\hline latitude & $36.9^{\circ} \mathrm{N}-37.3^{\circ} \mathrm{N}$ & $37.1^{\circ} \mathrm{N}-37.2^{\circ} \mathrm{N}$ & \\
\hline longitude & $4.4^{\circ} \mathrm{W}-4.8^{\circ} \mathrm{W}$ & $4.1^{\circ} \mathrm{W}-4.3^{\circ} \mathrm{W}$ & \\
\hline$b$ & $0.98 \pm 0.01$ & $1.06 \pm 0.03$ & \\
\hline$p$ & $0.84 \pm 0.01$ & $0.89 \pm 0.01$ & \\
\hline$D_{S}$ & $2.07 \pm 0.03$ & $2.08 \pm 0.03$ & \\
\hline
\end{tabular}

less attention has been paid to the distribution of distances between consecutive events. A power law, which derives from a truncated gamma distribution for small arguments, is assumed to model cumulative probabilities of both the elapsed times and distances. Additionally, the Weibull distribution and a logarithmic law have been also successful in some cases.

\section{Seismological and tectonic settings}

The southern part of the Iberian Peninsula is the most vulnerable to seismic risk in Spain. The area is situated in the central part of the Betic Cordillera (Fig. 1a). It includes the Granada Basin and several mountain ranges around it. The crust is characterised by thickness variations (GalindoZaldívar et al., 1997, 1999). The Granada Basin fault system created a set of blocks structured at different levels, thus allowing independent movements of them. These features fit into a general compressive framework, which produces contemporary extensional and compressive deformations on strike-slip faults. The seismogenetic areas are concentrated in three fracture systems having N10-30E, N30-60W and N70-100E directions. All the fracture systems are embedded in the Betic Area (Vidal, 1986; Peña et al., 1993; Posadas et al., 1993a, b) and the Granada Basin has a high activity of microearthquakes with hypocenters shallower than $20 \mathrm{~km}$.

The Andalusian Seismic Network installed by the Andalusian Institute of Geophysics provided a wide seismic catalogue constituted by 20000 events with extreme body- wave magnitudes reaching 5.5. According to the GutenbergRichter law (Fig. 1b), only events with magnitudes $M_{L} \geq 2.5$ have been considered, being 2.5 the minimum magnitude of completeness. This cut-off magnitude implies to finally dispose of a maximum number of 5648 events to be analysed. The study also includes the investigation of five seismic series, which locations are schematised in Fig. 2. These five seismic crises represent a varied sample of seismic activity and it should be interesting to observe if their interevent elapsed times and distances distribute with similar statistical parameters and functions. Some of the crises are characterised by a short duration (only three days) and others by a relatively long duration (more than 6 months). From the point of view of the magnitude range, some of the crises have a relatively wide range of available magnitudes (from 1.5 to 5.0), while others have narrower ranges, from 4.0 to 2.5.

1. The Adra-Berja area is located in the south-eastern Iberian Peninsula and it is characterised by a high level of seismicity; between December 1993 and March 1994, several hundred microearthquakes and earthquakes were recorded in this zone. The seismic series began with an earthquake of magnitude $M_{L}=5.0$ on 23 December and several aftershocks occurred, two of which reached $M_{L}=4.0$; a few days later, there was another earthquake of $M_{L}=4.9$.

2. In the Agrón seismogenetic region, several seismic crises have occurred in the 1985-2000 recording period. The main crisis began on 5 December with 

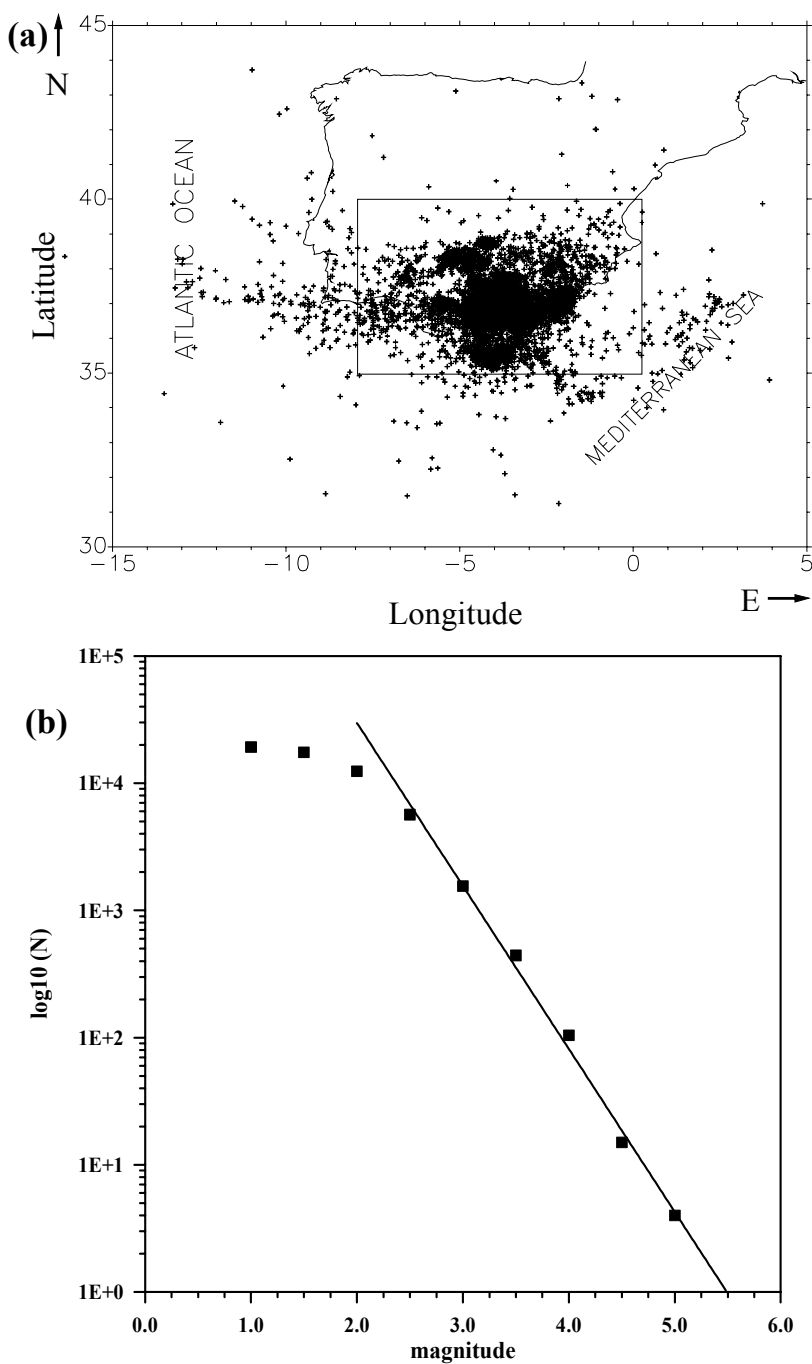

Fig. 1. (a) Area (inner rectangle) covered by the seismic activity in Southern Spain. (b) Gutenberg-Richter law for the seismic events within the inner rectangle. Magnitudes less than 2.5 depart remarkably from the cumulative law.

an earthquake of $M_{L}=4.0$ followed by another of $M_{L}=3$.9. Between 5 and 15 December 1988, 292 microearthquakes were recorded, with epicentres near the villages of Agrón and Cacín (Granada, Spain).

3. The Alborán Sea zone on the Mediterranean is close to that of Adra-Berja. The seismic series occurred from June 1997 until January 1998. After the beginning of the crisis with an event of $M_{L}=4.5$, more than $300 \mathrm{mi}$ croearthquakes were located in this area.

4. In the Antequera area only one seismic crisis occurred with a very short duration, between 7 and 9 June 1989 . At least 158 earthquakes of $M_{L}<3.4$ occurred.

5. The Loja region is an active zone where several seismic series have occurred, one of them in 1985, in a group of faults with orientation N40-55W. More than
Table 2. Parameters and least square regression coefficients for the power law (elapsed time and distances) and the Weibull distribution (elapsed times) when the whole seismic catalogue is considered. $d_{\max }$ and $t_{\max }$ represent the maximum distances an elapsed times predicted by the power law. Bold characters for $u$ and $K$ correspond to estimations achieved by maximum likelihood.

\begin{tabular}{ccccc}
\hline \multicolumn{5}{c}{ Power law } \\
$\delta D(\mathrm{~km})$ & $m=2.5$ & $m=3.0$ & $m=3.5$ & $m=4.0$ \\
\hline$a$ & 0.028 & 0.041 & 0.042 & 0.015 \\
$c$ & 0.598 & 0.535 & 0.512 & 0.660 \\
$\rho^{2}$ & 0.988 & 0.970 & 0.990 & 0.979 \\
$d_{\text {max }}$ & 395.1 & 391.7 & 488.61 & 580.1 \\
\hline \multicolumn{5}{c}{ Power law } \\
$\delta t$ (days) & $m=2.5$ & $m=3.0$ & $m=3.5$ & $m=4.0$ \\
\hline$a$ & 0.648 & 0.383 & 0.203 & 0.103 \\
$c$ & 0.504 & 0.433 & 0.456 & 0.453 \\
$\rho^{2}$ & 0.963 & 0.945 & 0.975 & 0.976 \\
$t_{\text {max }}$ & 2.36 & 9.17 & 33.01 & 151.11 \\
\hline \multicolumn{5}{c}{ Weibull distribution } \\
$\delta t$ (days) & $m=2.5$ & $m=3.0$ & $m=3.5$ & $m=4.0$ \\
\hline$u$ & 0.769 & 2.597 & 9.835 & 44.625 \\
$K$ & $\mathbf{0 . 7 6 9}$ & $\mathbf{2 . 4 9 5}$ & $\mathbf{9 . 5 3 2}$ & $\mathbf{4 2 . 2 8 0}$ \\
$K$ & 0.680 & 0.536 & 0.587 & 0.591 \\
$\rho^{2}$ & $\mathbf{0 . 6 9 7}$ & $\mathbf{0 . 6 0 3}$ & $\mathbf{0 . 6 6 1}$ & $\mathbf{0 . 7 0 9}$ \\
& 0.987 & 0.981 & 0.986 & 0.963 \\
\hline \multicolumn{5}{c}{}
\end{tabular}

300 microearthquakes and earthquakes were located in a very small area. The main event (3 February 1985) had $M_{L}=3.7$.

Table 1 summarizes the parameter $b$ of the GutenbergRichter law, the parameter $p$ of the Omori's law, the fractal dimension $D_{S}$ of the spatial earthquake distribution, the magnitude range $\Delta m$ for which completeness is assured by the Gutenberg-Richter law, the number of events, latitude and longitude range and recording period $\Delta t$ of every seismic crisis. All these details are compiled from Posadas et al. (2002), who tested the reliability of the parameters $b, p$ and $D_{S}$. The spatial clustering was estimated by a standard box-counting and the computation of the Morishita's index (Ouchi and Uekawa, 1986). It has to be pointed out that due to the close monitoring of these seismic crises by the IAG, the completeness of the seismic series is sometimes reasonably assured for magnitudes below 2.5. In this way, a low magnitude as 1.5 in the cases of Adra and Agrón has been accepted after the verification of the Gutenberg-Richter law for these crises. 


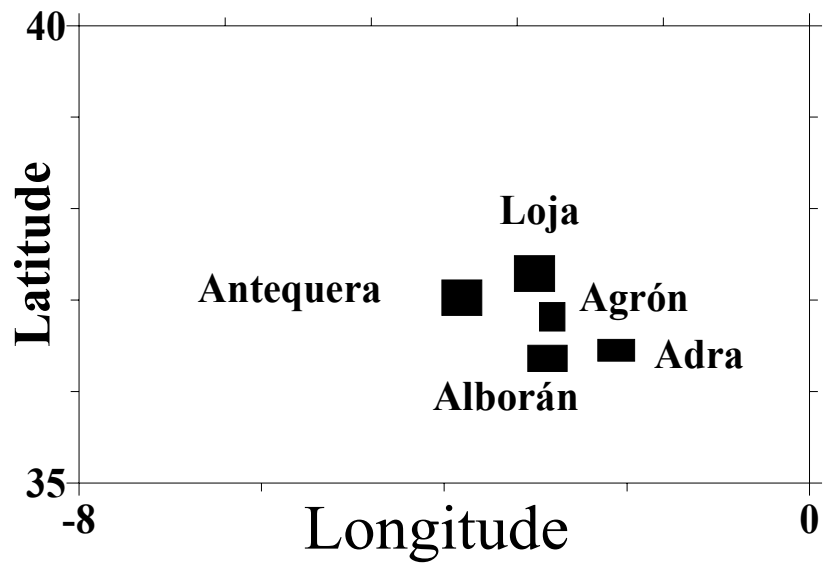

Fig. 2. Emplacement of the five seismic crises in Southern Spain.

\section{Results}

\subsection{Consecutive events for the whole catalogue}

The set of elapsed times, $\delta T$, and distances, $\delta D$, between consecutive seismic events have been analysed for four different threshold magnitudes of 2.5, 3.0, 3.5 and 4.0. These threshold values reduce the number of available events to 5648, 1951, 441 and 103, respectively. The first threshold value is the lowest magnitude for which the GutenbergRichter law depicts completeness (Fig. 1b), while the last one is the maximum magnitude for which the series of $\delta T$ and $\delta D$ are long enough to obtain reliable empiric cumulative probabilities.

For the series of $\delta T$ both a power law

$P\left(x_{0} \leq x\right)=a x^{c}$

and the Weibull distribution function

$P\left(x_{0} \leq x\right)=1-\exp \left\{-\left(\frac{x-\varepsilon}{u-\varepsilon}\right)^{K}\right\} x \geq \varepsilon ; u, K>0$

are assumed. Besides its first applications in the analysis of fatigue of materials, the Weibull distribution has been often used in stochastic processes where the analysis of the elapsed time between certain events is investigated. As an example, two applications in different fields of the Earth's sciences such as seismology (Correig et al., 1997) and climatology (Burgueño et al., 2004) can be cited. Other distributions easier to manage, as the exponential function, could be also considered. Nevertheless, after some tests, the best fit was always performed by the power law and the Weibull distribution. It has to be remembered that the Weibull distribution includes as a particular case $(K=1)$ the exponential function.

The power law is very simple and characterise the fractal behaviour of seismicity. The parameters $a$ and $c$ of this law can be easily determined by means of a least square regression in $\log -\log$ scales. The parameter $\varepsilon$ of the Weibull function is set equal to zero, which is equivalent to assume that
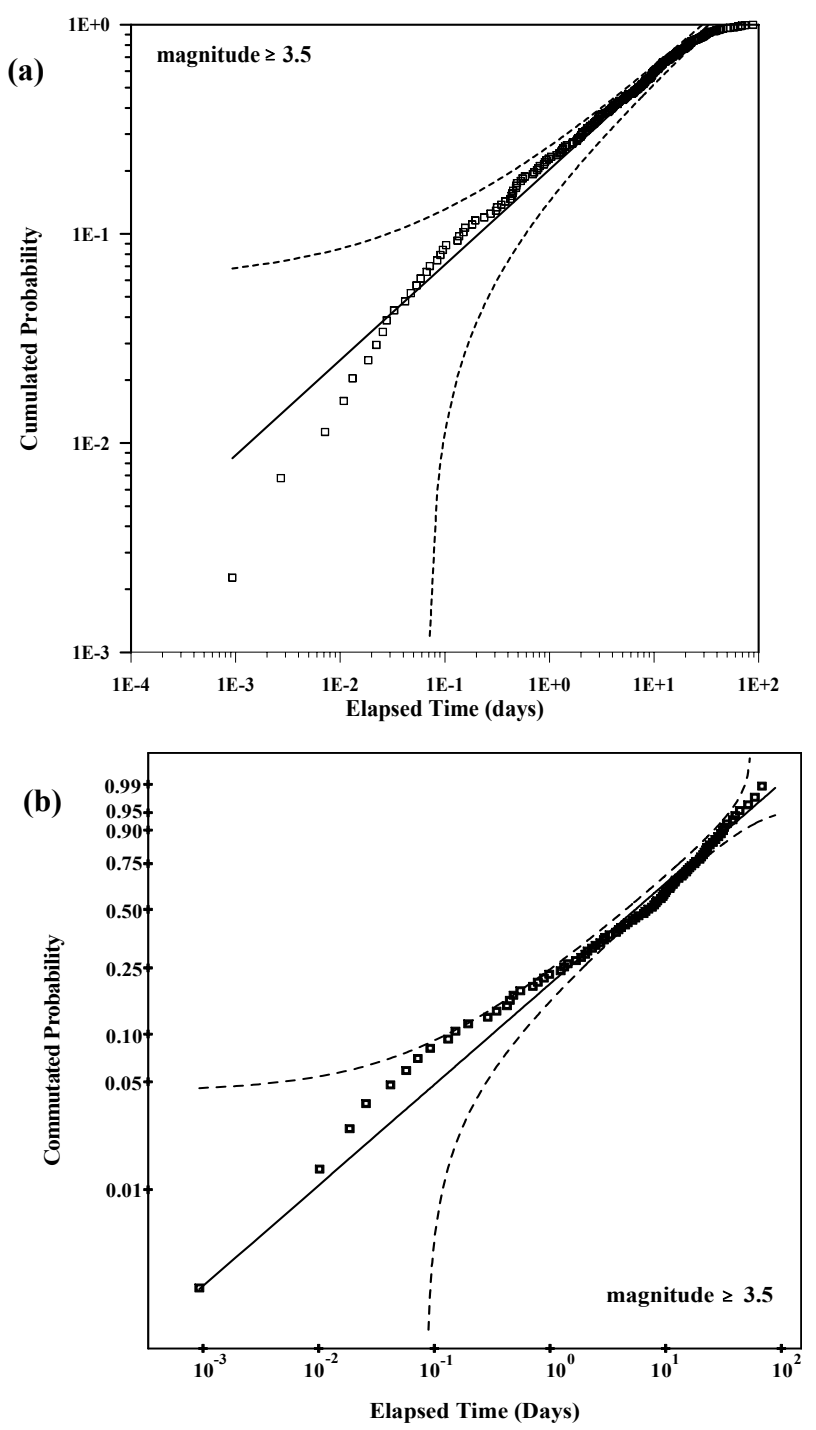

Fig. 3. Power law (a) and Weibull distribution (b) for the elapsed time between consecutive events for a threshold magnitude of 3.5 .

the minimum elapsed time is almost null in comparison with the total length of the seismic episode, and parameters $u$ and $K$ can be easily estimated in a first approach by a least square regression in a log-log scale. Empirical and theoretical models are represented in terms of $\log \{-\log (1-p)\}$ versus $\log (x)$ for a first visual inspection of the possible linearity, since, in these scales, data accomplishing the Weibull model are evidenced by a linear behaviour. Better estimations of $u$ and $K$ are achieved by maximum likelihood and the KolmogorovSmirnov test (Lemeshko and Postovalov, 2001) in the case of composite hypothesis can be considered in order to decide if the Weibull distribution performs well empiric data. The cumulative probabilities for the series $\delta D$ of distances are also modelled by the power law (Eq. 1).

Table 2 shows the values of the parameters and the corresponding least square regression coefficients $\rho^{2}$ for series $\delta T$ and $\delta D$ and the maximum elapsed times and distances 
Table 3. Parameters and least square regression coefficients for the power and logarithmic laws and the Weibull distribution in the case of elapsed times computed from the starting (main) event. $t_{\max }$ (days) represents the maximum time predicted by the logarithmic laws. $\delta T$ is the time interval for which every model fits well empiric data. Bold characters for $u$ and $K$ correspond to estimations achieved by maximum likelihood.

\begin{tabular}{|c|c|c|c|c|c|c|c|c|c|}
\hline \multirow[b]{2}{*}{ Crisis } & \multicolumn{4}{|c|}{ Power law } & \multicolumn{5}{|c|}{ Logarithmic law } \\
\hline & $a$ & $c$ & $\rho^{2}$ & $\delta T$ & $\alpha$ & $\beta$ & $\rho^{2}$ & $t_{\max }$ & $\delta T$ \\
\hline Adra & 11.49 & 1.012 & 0.985 & $0-0.02$ & 0.992 & 0.196 & 0.958 & 1.1 & $0.02-7.0$ \\
\hline Agrón & 2.380 & 0.502 & 0.913 & $0-0.02$ & 1.062 & 0.135 & 0.959 & 0.6 & $0.02-0.9$ \\
\hline Alborán & 0.581 & 0.287 & 0.946 & $0-0.02$ & 0.638 & 0.103 & 0.991 & 33.6 & $0.02-20.0$ \\
\hline Antequera & 4.019 & 0.504 & 0.922 & $0-0.02$ & 1.148 & 0.117 & 0.957 & 0.3 & $0.02-0.3$ \\
\hline Loja & 1.008 & 0.342 & 0.867 & $0-0.02$ & 0.852 & 0.107 & 0.896 & 4.0 & $0.02-20.0$ \\
\hline Crisis & $u$ & $\begin{array}{c}\text { Weibull } \\
\quad K\end{array}$ & $\rho^{2}$ & & & & & & \\
\hline Adra & $\begin{array}{l}0.157 \\
\mathbf{0 . 1 6 7}\end{array}$ & $\begin{array}{l}0.773 \\
\mathbf{0 . 6 9 8}\end{array}$ & 0.988 & & & & & & \\
\hline Agrón & $\begin{array}{l}0.056 \\
\mathbf{0 . 0 6 1}\end{array}$ & $\begin{array}{l}0.698 \\
\mathbf{0 . 6 0 5}\end{array}$ & 0.972 & & & & & & \\
\hline Alborán & $\begin{array}{l}0.933 \\
\mathbf{1 . 0 4 6}\end{array}$ & $\begin{array}{l}0.392 \\
\mathbf{0 . 3 9 6}\end{array}$ & 0.982 & & & & & & \\
\hline Antequera & $\begin{array}{l}0.020 \\
\mathbf{0 . 0 2 3}\end{array}$ & $\begin{array}{l}0.699 \\
\mathbf{0 . 5 9 8}\end{array}$ & 0.976 & & & & & & \\
\hline Loja & $\begin{array}{l}0.177 \\
\mathbf{0 . 2 0 7}\end{array}$ & $\begin{array}{l}0.486 \\
\mathbf{0 . 3 8 9}\end{array}$ & 0.944 & & & & & & \\
\hline
\end{tabular}

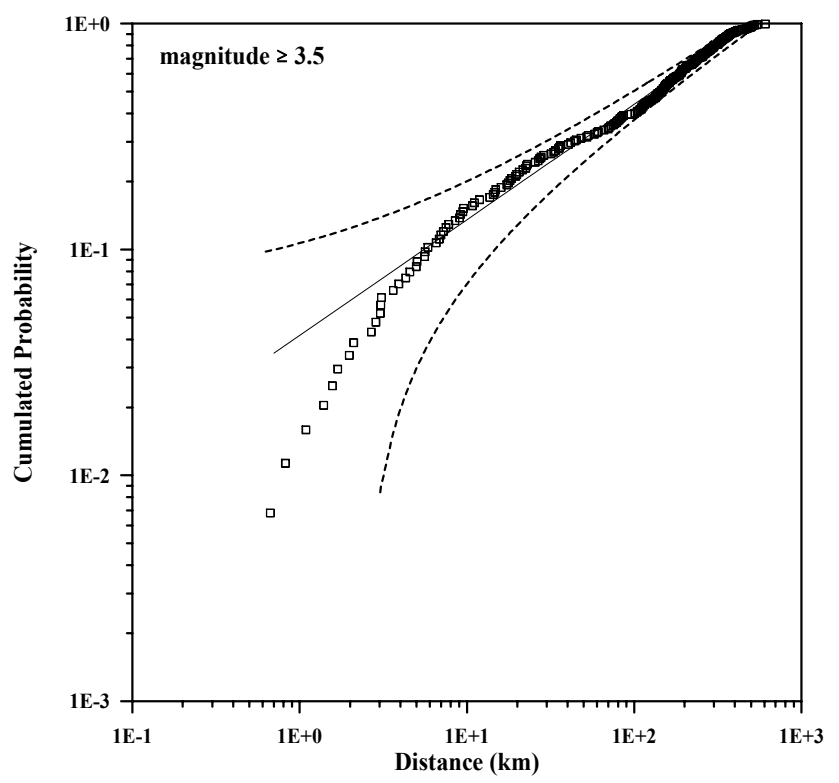

Fig. 4. Power law for the distance between consecutive events for a threshold magnitude of 3.5. Dashed lines correspond to $95 \%$ Kolmogorov-Smirnov uncertainty bands derived with the assumption of simple hypothesis.

derived from the power law (Eq. 1). Bold characters correspond to parameters $u$ and $K$ estimated by maximum likelihood. Figure 3 shows the fit performed by the power law (Eq. 1) and the Weibull distribution (Eq. 2) for the series $\delta T$ and a threshold magnitude of 3.5. In the case of the Weibull distribution (Fig. 3b), the straight line depicts the theoretical distribution computed from parameters $u$ and $K$ derived by means of maximum likelihood and dashed lines the $95 \%$ Kolmogorov-Smirnov uncertainty bands for composite hypothesis, which can be roughly estimated by $0.91 / n^{1 / 2}, n$ being the number of elements of the series. Dashed lines in Fig. 3 a corresponds to the $95 \%$ Kolmogorov-Smirnov uncertainty bands for simple hypothesis (Benjamin and Cornell, 1970), roughly estimated by $1.36 / n^{1 / 2}$. Figure 4 shows the fit performed by the power law (Eq. 1) for the series of distances and for the same threshold magnitude. Dashed lines of this figure are similar to those corresponding to Fig. 3a.

\subsection{Events belonging to a seismic crisis}

In relation to the seismic crises described in Sect. 2, two types of series are considered: 1) interevent elapsed times and distances; 2) elapsed times and distances from the first (main) shock. Tables 3 to 6 summarise the values of the parameters and least square regression coefficients for series $\delta T$ and $\delta D$, both taking as reference the previous event of the crisis and the mainshock of the seismic sequence. In some cases, a combination of the power law (Eq. 1) and the logarithmic law

$P\left(x_{0} \leq x\right)=\alpha+\beta \log (x)$

improves the modelling of the empiric distribution of distances and elapsed times. The parameters $\alpha$ and $\beta$ can be easily determined by a least square regression in a linear-log 
(a)
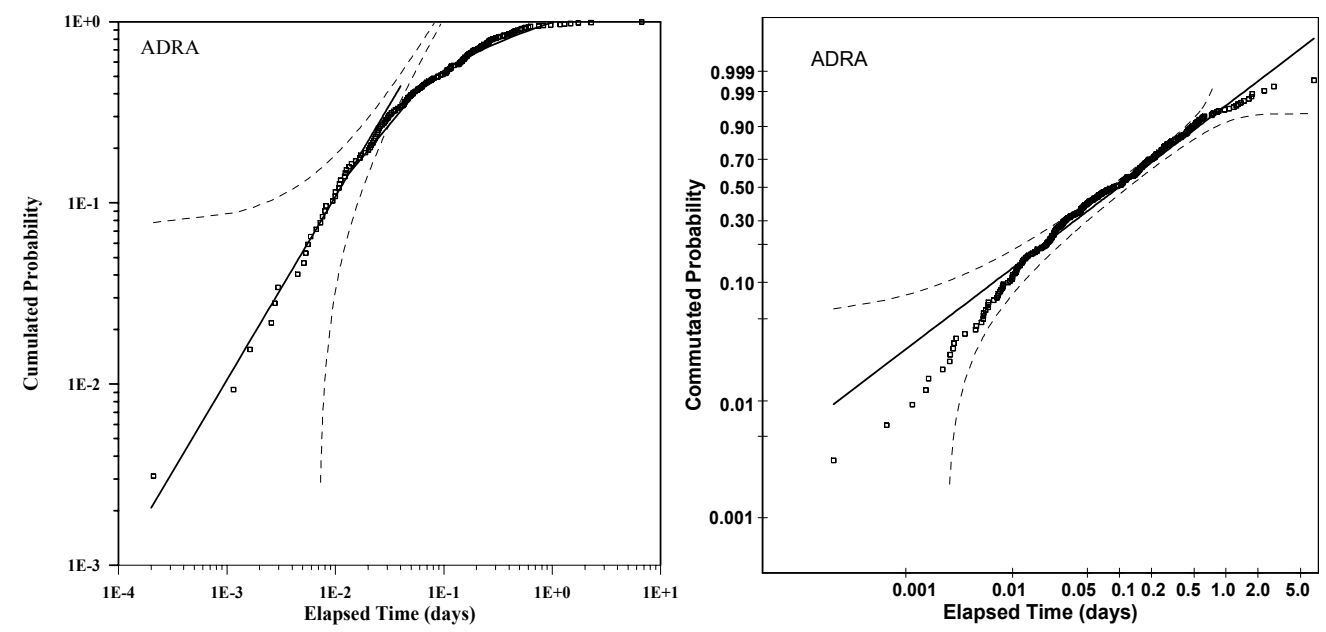

(b)
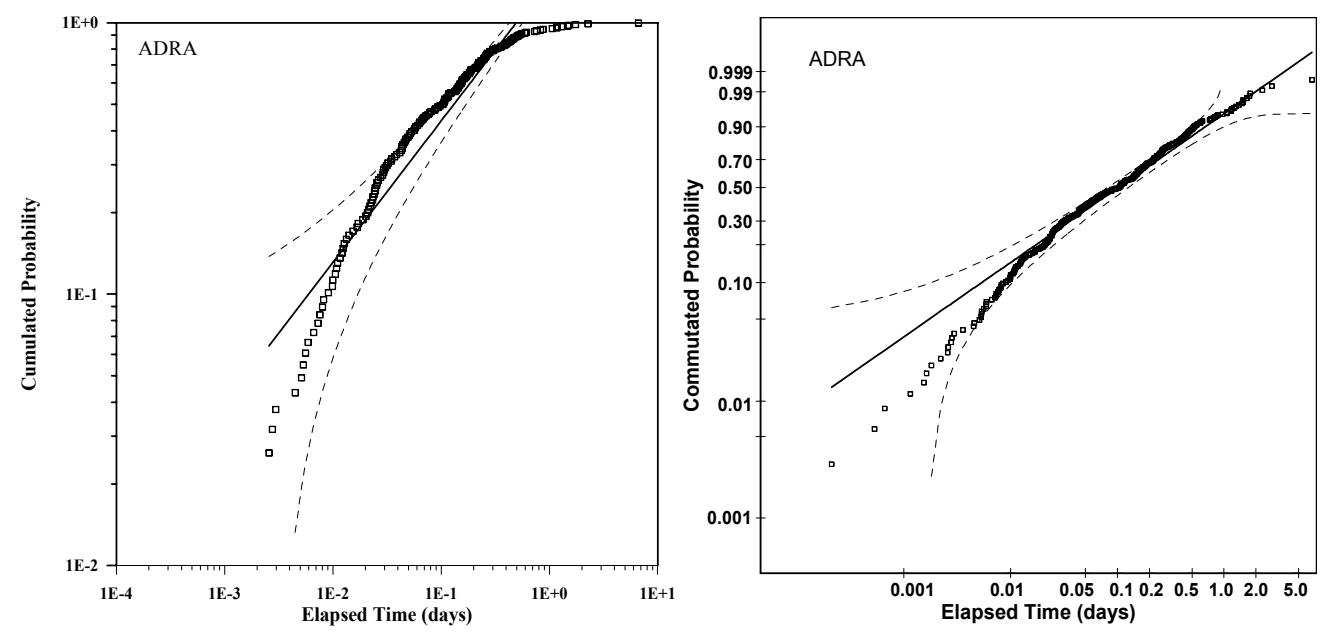

(c)

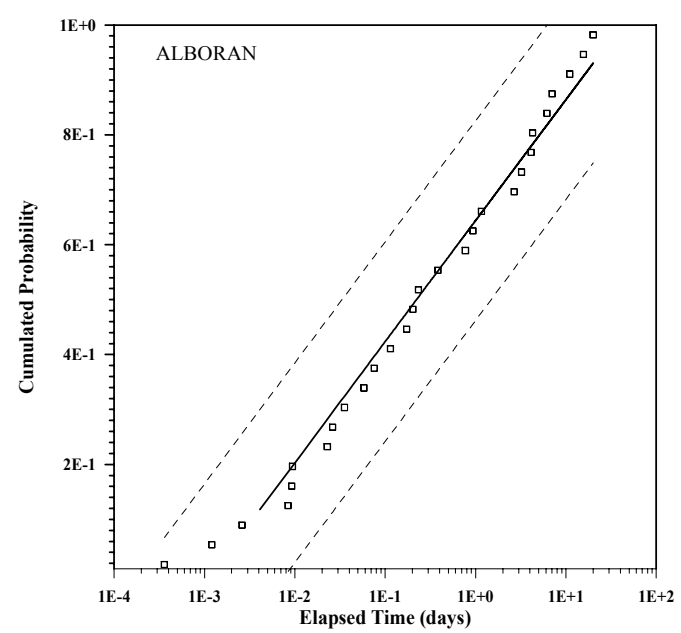

Fig. 5. Power law and Weibull distribution for elapsed times of aftershocks referred to the main event (a) and between consecutive aftershocks (b), both in the case of Adra. The case of Alborán for consecutive aftershocks (c) is well performed by the logarithmic law. 
Table 4. Parameters and least square regression coefficients for the power and logarithmic laws and the Weibull distribution of the elapsed times in the case of consecutive events of a seismic crisis. $t_{\max }$ (days) represents the maximum time predicted by the logarithmic law. $\delta T$ is the time interval for which every model fits well empiric data. Bold characters for $u$ and $K$ correspond to estimations achieved by maximum likelihood.

\begin{tabular}{|c|c|c|c|c|c|c|c|c|c|}
\hline \multirow[b]{2}{*}{ Crisis } & \multicolumn{4}{|c|}{ Power law } & \multicolumn{5}{|c|}{ Logarithmic law } \\
\hline & $a$ & $c$ & $\rho^{2}$ & $\delta T$ & $\alpha$ & $\beta$ & $\rho^{2}$ & $t_{\max }$ & $\delta T$ \\
\hline Adra & 4.765 & 0.825 & 0.980 & $0-0.07$ & 0.955 & 0.177 & 0.902 & 1.3 & $0.07-8.0$ \\
\hline Agrón & 6.294 & 0.678 & 0.950 & $0-0.05$ & 1.030 & 0.111 & 0.952 & 0.8 & $0.05-1.0$ \\
\hline Alborán & & & & & 0.644 & 0.104 & 0.993 & 30.7 & $0-20.0$ \\
\hline Antequera & 6.891 & 0.569 & 0.981 & $0-0.03$ & 1.080 & 0.083 & 0.937 & 0.4 & $0.03-0.4$ \\
\hline Loja & 3.764 & 0.627 & 0.973 & $0-0.04$ & 0.824 & 0.098 & 0.904 & 6.1 & $0.04-20.0$ \\
\hline Crisis & $u$ & $\begin{array}{c}\text { Weibull } \\
\quad K\end{array}$ & $\rho^{2}$ & & & & & & \\
\hline Adra & $\begin{array}{l}0.170 \\
\mathbf{0 . 1 8 1}\end{array}$ & $\begin{array}{l}0.751 \\
0.639\end{array}$ & 0.990 & & & & & & \\
\hline Agrón & $\begin{array}{l}0.056 \\
\mathbf{0 . 0 6 1}\end{array}$ & $\begin{array}{l}0.701 \\
\mathbf{0 . 6 0 3}\end{array}$ & 0.975 & & & & & & \\
\hline Alborán & $\begin{array}{l}0.938 \\
\mathbf{1 . 0 4 7}\end{array}$ & $\begin{array}{l}0.389 \\
\mathbf{0 . 3 9 5}\end{array}$ & 0.985 & & & & & & \\
\hline Antequera & $\begin{array}{l}0.020 \\
\mathbf{0 . 0 2 3}\end{array}$ & $\begin{array}{l}0.701 \\
\mathbf{0 . 5 9 9}\end{array}$ & 0.975 & & & & & & \\
\hline Loja & $\begin{array}{l}0.196 \\
\mathbf{0 . 2 2 6}\end{array}$ & $\begin{array}{l}0.507 \\
\mathbf{0 . 4 1 3}\end{array}$ & 0.962 & & & & & & \\
\hline
\end{tabular}

Table 5. Parameters and least square regression coefficients for power and logarithmic laws in the case of distances from the mainshock to every aftershock. $d_{\max }$ (days) represents the maximum distance predicted by the logarithmic law, except for the case of Agrón, for which a power law performs well all available data. $\delta d$ is the distance interval for which every model fits well empiric data.

\begin{tabular}{|c|c|c|c|c|c|c|c|c|c|c|}
\hline \multirow[b]{2}{*}{ Crisis } & \multicolumn{5}{|c|}{ Power } & \multicolumn{5}{|c|}{ Logarithmic } \\
\hline & $a$ & $b$ & $\rho^{2}$ & $d_{\max }$ & $\delta d$ & $\alpha$ & $\beta$ & $\rho^{2}$ & $d_{\max }$ & $\delta d$ \\
\hline Adra & 0.001 & 2.311 & 0.985 & & $2.0-20.0$ & 0.389 & 0.156 & 0.890 & 50.2 & $20.0-50.0$ \\
\hline Agrón & 0.364 & 1.290 & 0.960 & 2.2 & $0.1-5.0$ & & & & & \\
\hline Alborán & 0.004 & 2.031 & 0.967 & & $3.0-15.0$ & 0.463 & 0.142 & 0.916 & 43.9 & $15.0-50.0$ \\
\hline Antequera & & & & & & -0.280 & 0.465 & 0.974 & 15.7 & $1.0-15.0$ \\
\hline Loja & 0.057 & 1.456 & 0.987 & & $1.0-4.0$ & -0.090 & 0.411 & 0.988 & 14.2 & $4.0-22.0$ \\
\hline
\end{tabular}

scale. In a few cases, the best fit is given either by the power or by the logarithmic law and the combination of both models does not perform better. As in Table 2, maximum elapsed times and distances deduced either from Eq. (1) or Eq. (2) are included in Tables 3 to 6 and bold characters for $u$ and $K$ correspond to estimations derived by maximum likelihood.

As an example, Fig. 5 shows the elapsed times from the mainshock and the interevent elapsed times for the seismic crisis of Adra, with the power law and the Weibull distribution. The good fit of the logarithmic law for the Alborán crisis interevent elapsed times is also shown. 95\% uncertainty bands have been estimated in terms of composite hypothesis for the Weibull distribution. For the other three cases, 95\% uncertainty bands derived from the simple hypothesis have been represented. Figure 6 illustrates two examples of the series of distances, as interevent distances and distances from the mainshock. 95\% uncertainty bands correspond to the Kolmogorov-Smirnov test for simple hypothesis. If the power law is assumed for the Adra seismic crisis, the law itself manifests the necessity of a complementary logarithmic law to explain the distribution for the largest distances and elapsed times. For instance, empirical data depart significantly from the power law for distances exceeding approximately $10 \mathrm{~km}$ (distances to mainshock) and $5 \mathrm{~km}$ (interevent distances).

\section{Discussion and conclusions}

Some previous considerations have to be introduced for a right interpretation of the results. The first point concerns with the fact that the most robust estimation of the Weibull 
Table 6. Parameters and least square regression coefficients for power and logarithmic laws in the case of distances between consecutive aftershocks of a seismic crisis. $d_{\max }$ (days) represents the maximum distance predicted by the power law, excepts for Adra and Loja, which need for a combination with a logarithmic law. $\delta d$ is the distance interval for which every model fits well empiric data.

\begin{tabular}{ccccccccccc}
\hline & \multicolumn{9}{c}{ Power } & \multicolumn{8}{c}{ Logarithmic } \\
Crisis & $a$ & $b$ & $\rho^{2}$ & $d_{\max }$ & $\delta d$ & $\alpha$ & $\beta$ & $\rho^{2}$ & $d_{\max }$ & $\delta d$ \\
\hline Adra & 0.038 & 1.345 & 0.979 & & $0.2-2.0$ & -0.105 & 0.308 & 0.989 & 36.1 & $2.0-50.5$ \\
Agrón & 0.230 & 1.228 & 0.924 & 3.4 & $0.1-9.0$ & & & & & \\
Alborán & 0.063 & 0.887 & 0.954 & 22.6 & $0.4-5.0$ & & & & & \\
Antequera & 0.049 & 1.174 & 0.967 & 13.1 & $0.6-16.0$ & & & & & \\
Loja & 0.028 & 1.579 & 0.951 & & $0.5-3.0$ & -0.408 & 0.502 & 0.981 & 16.5 & $3.0-20.0$ \\
\hline
\end{tabular}

distribution parameters is that based on the maximum likelihood. In spite of quite good values of the least square regression coefficient, the minimization process in a $\log (1-\log (P))$ scale leads to small deviations for small elapsed times and strong deviations for intermediate elapsed times. Second, parameters for the power and logarithmic laws can be assumed as derived from graphical analysis (probability paper representations), which lead to linear graphs in log-log and linear$\log$ scales. In these cases, the least square regression coefficient has to be carefully considered because it is not again the best factor to decide the vicinity of empiric and theoretical distributions. One more time differences between theoretical and empiric distribution are not directly minimised. A third point concerns the uncertainty bands derived from the simple and composite Kolmogorov-Smirnov test hypothesis. For the case of the Weibull distribution, Kolmogotrov-Smirnov test with composite hypothesis have to be used, given that the same empiric data is used for the test and for the parameter estimation. Nevertheless, this kind of test seems to be unknown for the power and logarithmic laws. Consequently, simple hypothesis bands have to be assumed as a criterion to decide if power and logarithmic laws are rejeced. Given that these bands can be assumed wider than those derived from composite hypothesis, empirical distributions within simple hypothesis bands are not a guarantee that a power or logarithmic law is the best representation of the distribution of elapsed times or distances. Graphical analyses, least square regression and simple hypothesis bands only suggest that these laws could be a right model.

The analysis of the whole catalogue shows that the power law reasonably explains the empirical distribution of distances and elapsed times when the threshold magnitude varies. In spite of the fit is not perfect, it is much better than those observed for the seismic crises. It has to be remembered that, when fitting power laws, it is quite usual to detect discrepancies between empiric data and the theoretical power function for values of the cumulative probability very close to 1.0. For example, it can be observed in Fig. 3a empirical data out of the Kolmogorov-Smirnov simple band only for cumulative probabilities exceeding 0.9. Therefore, it could be assumed a universal law for both quantities and some kind of fractal structure in reason to the mathematical character of this law. In fact, by following classic definitions of fractal dimension, such as those associated with rescaled analysis, clustering dimension or correlation dimension (Grassberger and Procaccia, 1983a, b; Korvin, 1992; Dicks, 1999), the parameter $c$, ranging from 0.4 to 0.7 , should be considered the fractal dimension of the series analysed. The Weibull distribution for elapsed times also fits quite well the empiric data. Nevertheless, empiric data are sometimes out of the 95 uncertainty bands for some inter-event time interval, as Fig. 3b manifests.

The analysis of the five seismic crises shows a more complex behaviour and suggests the breakdown of the fractal behaviour for all cases where a power law is not sufficient to perform empiric data. When the elapsed times are computed from the first (main) shock, the best fit is furnished by the combination of power and logarithmic laws and the transition from one law to another is observed close to 0.02 days in all cases. It is worth of mention that, opposite to the case of the whole catalogue, elapsed times of the order of 0.02 days are not associated with cumulative probabilities close to 1.0. As an example, the case of Adra crisis (Fig. 5a) is characterised for the necessity of changing to a logarithmic behaviour for cumulative probabilities exceeding 0.3 . This elapsed time of transition would be the value from which the approach of the cumulative gamma distribution to a power law (Arfken, 1985; Abramowitz and Stegun, 1970) becomes inaccurate. Once more, the Weibull distribution offers a similar satisfactory fit for empirical series, when comparing with the other two laws. Nevertheless, the Loja crisis seems to be much better reproduced by the Weibull distribution than by the combined power and logarithmic laws.

For the interevent times, in some cases the fit with one law performs better (see for example the case of the Alborán crisis, which is very well-fitted by the logarithmic law). When the combined laws are necessary, the elapsed time of transition (0.03-0.7 days) is quite similar to that obtained for times referred to mainshock. Once more, the Weibull distribution offers quite similar fits. Moreover, assuming that, as in the case of the whole catalogue, parameter $c$ could represent the fractal dimension of the series, estimated now by only considering the range for which the power law performs well, it is observed that this dimension does not change remarkably 
from one crisis to another. Nevertheless, notable changes are detected when comparing cases corresponding to times from mainshock and interevent times. Additionally, it is worth of mention that only fractal dimensions for Adra, according to Tables 3 and 4, are notably different to those obtained for the whole catalogue and the four threshold magnitudes.

Concerning the analysis of distances, when they are computed from the location of the mainshock, the combination of power and logarithmic laws is not always necessary and the transition from one law to another varies remarkably from 4.0 to $20.0 \mathrm{~km}$. The series of distances for Antequera crisis is well performed by only a logarithmic law and Agrón series by only a power law. When distances are computed between consecutive aftershocks, the logarithmic law is not necessary for three crises (Agrón, Alborán, Antequera) and fits offered by the power law are reasonably good. Due to only two examples can be revised, nothing can be safely stated with respect to distances for which the transition from power to logarithmic laws is observed. With respect to the fractal dimension, the parameter $c$ for the whole catalogue reaches values $(0.5$ to 0.7$)$ clearly lower than those derived for the seismic crises. Additionally, whereas $c$ ranges from 0.9 to 1.6 for distances between consecutive aftershocks, the fractal dimension increases up to 1.3-2.3 for distances referred to the mainshock.

In short, different behaviours have been observed for series derived from the whole seismic catalogue and from subsets of seismic crises. Nevertheless, a single power law with changing parameters could explain more or less satisfactorily in some cases the distribution of elapsed times and distances. The most relevant difference is the necessity of a logarithmic law to complement elapsed time and distance distributions derived from seismic crises. The elapsed time for which the change from the power to the logarithmic law takes place is always within a narrow time interval from 0.02 to 0.07 days. In this way, a different behaviour in the seismic crises for relatively short and long waiting times could be assumed. This behaviour in the case of distances could be in some way justifiable if we imagine a rough division between aftershocks near or away from the mainshock. Unfortunately, nothing can be safely stated concerning the transition from power to logarithmic laws for the case of seismic crises and distance distributions. In the case of elapsed times, it seems to be sometimes a distinction between aftershocks close and remote in time to the mainshock.

In spite of the necessity of an upper bound for the argument of the power and logarithmic laws, this fact should not be a shortcoming. Firstly, there is a maximum empirical distance imposed by the geographical extension covered by the seismic crisis or the whole catalogue. Secondly, it is meaningless to expect elapsed times tending to infinite for the whole catalogue. It would imply the finish of the activity in the seismotectonic area covered by the catalogue. Only in the case of the seismic crises, it could be partially justified in terms of the time decay of aftershocks, quantified by the Omori's law. From this point of view, although the Weibull distribution for elapsed times would be sometimes a better
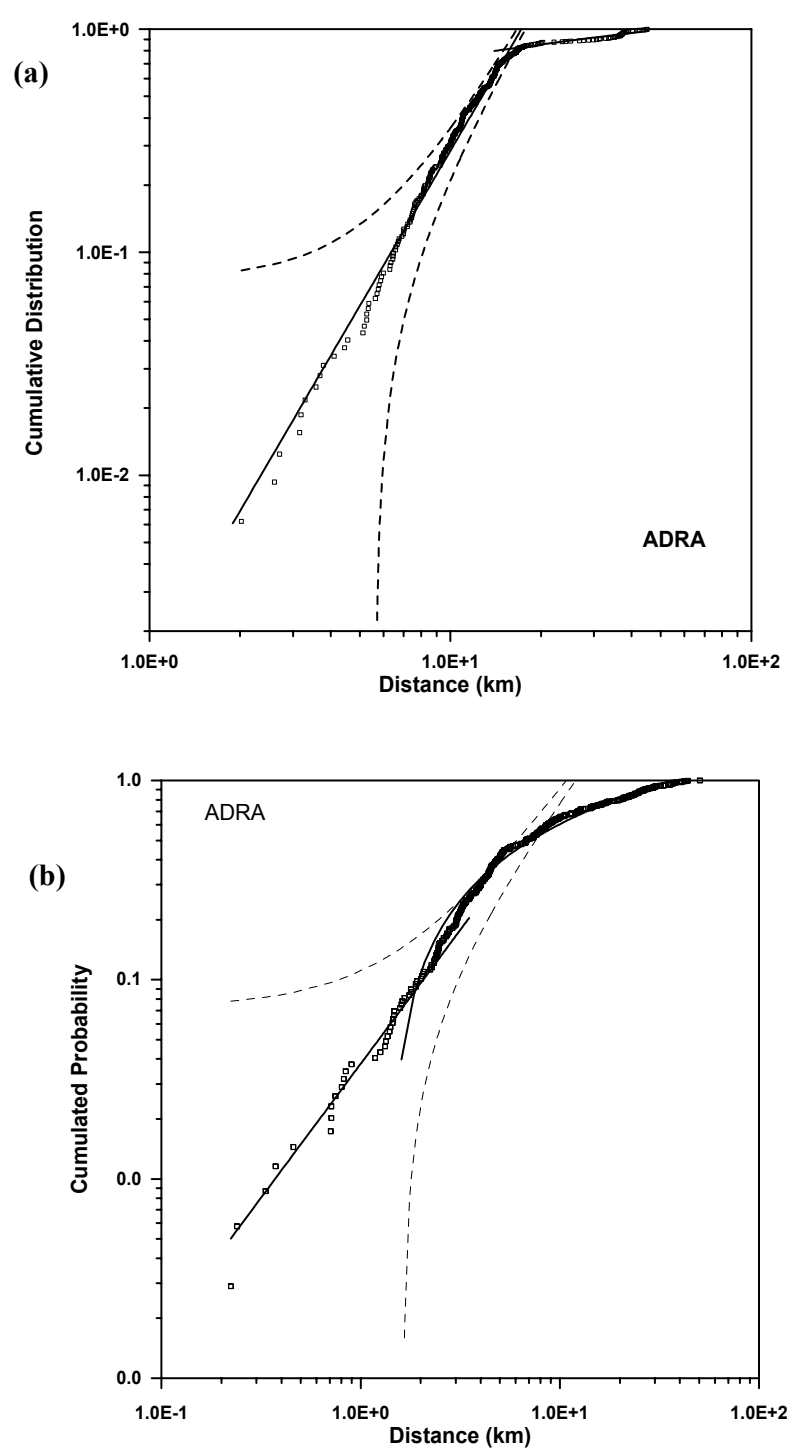

Fig. 6. Distribution of distances from the mainshock to the aftershocks (a) and distances between consecutive aftershocks (b).

statistical option than the power law, a shortcoming should be that of an argument extended to infinite.

Finally, the set of results concerning functions used to modelling the elapsed time and distances between seismic events have to be considered as a contribution to the theory of continuous time random walks (CTRW), which is usually described in terms of Lévy distribution functions and tries to explain distribution of waiting times and localisation of hypocenters in a seismotectonic region. The results derived here for the Southern Iberian Peninsula should be considered for future CTRW models concerning space-time diffusion of the seismic activity. It could be especially useful for the analysis of the whole seismic activity of Southern Iberia, due to in this case simple power laws seem to be sufficient for a quite good description of waiting times and distances. The case of the seismic crises is a bit more complex, due to sometimes combined power and logarithmic laws are necessary. 
Acknowledgements. This research was supported by the Spanish Ministry of Science and Technology through the projects REN2001-2418-C04-01 RIES and REN2003-07170 RIES.

Edited by: C. Goltz

Reviewed by: T. Chelidze, V. I. German and another referee

\section{References}

Abramowitz, M. and Stegun, I. A.: Handbook of Mathematical Functions, Dover Publications Inc., New York, 1046, 1970.

Arfken, G.: Mathematical Methods for Physicists (Third Edition), Academic Press Inc., San Diego, California, 985, 1985.

Benjamin, J. R. and Cornell, C. A.: Probability, statistics and decision for civil engineers, McGraw-Hill, New York, 685, 1970.

Bouchaud, J. P. and Georges, A.: Anomalous diffusion in disordered media: Statistical mechanism, models and physical applications, Physics Reports, 195, 4-5, 127-293, 1990.

Burgueño, A., Serra, C., and Lana, X.: Monthly and annual statistical distributions of the daily rainfall at the Fabra Observatory (Barcelona, NE Spain) for the years 1917-1999, Theor. Appl. Climatol., 77, 57-75, 2004.

Chen, Y., Chen, L., Liu, Z., and Wu, R.: A new fractal approach to the clustering of earthquakes: Physical fractal, Bull. Seism. Soc. Am., 88, 89-94, 1998.

Corral, A.: Time-decreasing hazard and increasing time until the next earthquake, Condensed Matter, 0310407, 2003.

Corral, A.: Long term clustering, scaling and universality in the temporal occurrence of earthquakes, Phys. Rev. Lett., 92, 10, 108501/1-4, 2004

Correig, A. M., Urquizú, M., Vila, J., and Martí, J.: Analysis of the temporal occurrence of seismicity at Deception Island (Antartica), A non-linear approach, Pure Appl. Geophys., 149, 553 $574,1997$.

Dicks, C.: Nonlinear time series analysis. Nonlinear Time Series and Chaos (4), World Scientific, London, 209, 1999.

Feller, W.: An introduction to Probability Theory and Applications, Wiley, New York, 2, 1966.

Galindo-Zaldívar, J., Jabaloy, A., González-Lodeiro, F., and Aldaya, F.: Crustal structure of the central sector of the Betic Cordillera (SE Spain), Tectonics, 16, 18-37, 1997.

Galindo-Zaldivar, J., Jabaloy, A., Serrano, I., Morales, J., GonzalezLodeiro, F., and Torcal, F.: Recent and present-day stresses in the Granada basin (Betic Cordilleras: Example of a late Miocenepresent-day extensional basin in a convergent plate boundary, Tectonics, 18, 686-702, 1999.

Gnedenko, B. V. and Kolmogorov, A. N.: Limit Distributions for sums of independent random variables, Addison Wesley Reading, MA, 1954.

Goltz, C.: Fractal and Chaotic Properties of Earthquakes, Springer, Berlin, 205, 1998.

Grassberger, P. and Procaccia, I.: Characterization of strange attractors, Phys. Rev. Lett., 50, 346-349, 1983a.
Grassberger, P. and Procaccia, I.: Estimation of the Kolmogorov entropy from a chaotic signal, Phys. Rev. (A), 28, 2591, 1983 b.

Korvin, G.: Fractal models in the Earth sciences, Elsevier, Amsterdam, 1992.

Lemeshko, B. Yu. and Postovalov, S. N.: Application of the non parametric goodness-of-fit tests in testing composite hypothesis. Optoelectronics, Instrumentation and Data Processing, 2, 76-88, 2001.

Matcharashvili, T., Chelidze, T., and Javakhishvili, Z.: Nonlinear analysis of magnitude and interevent time interval sequences for earthquakes of the Caucasian region, Nonlin. Proc. Geophys., 7, 9-19, 2000,

SRef-ID: 1607-7946/npg/2000-7-9.

Matcharashvili, T., Chelidze, T., Javakhishvili, Z., and Ghlonti, E.: Detecting differences in temporal distribution of small earthquakes before and after large events, Computers \& Geosciences, 28, 693-700, 2002.

Ouchi, T. and Uekawa, T.: Statistical analysis of the spatial distribution of earthquakes before and after large earthquakes, Phys. Earth Planet. Int., 44, 211-225, 1986.

Peña, J., Vidal, F., Posadas, A., Morales, J., Alguacil, G., De Miguel, F., Ibáñez, J., Romacho, M., and López-Linares, A.: Space clustering properties of the Betic-Alboran earthquakes in the period 1962-1989, Tectonophysics, 221, 125-134, 1993.

Posadas, A. M., Vidal, F., De Miguel, F., Alguacil, G., Peña, J., Ibañez, J. M., and Morales, J.: Spatial-temporal analysis of a seismic series using the Principal Components Method, The Antequera Series (Spain), 1989, J. Geophys. Res., 98, 1923-1932, 1993a.

Posadas, A. M., Vidal, F., Morales, J., Peña, J. A., Ibañez, J., and Luzon, F.: "Spatial and temporal analysis of a seismic series using a new version of three point method, Application to Antequera (Spain) 1989 earthquakes, Phys. Earth Planet. Int., 80, $159-168,1993 b$

Posadas, A. M., Morales, J., Vidal, F., Sotolongo-Costa, O., and Antoranz, J. C.: Continuous Time Random Walks and Seismic Series: Southern Spain Seismic Series and Lévy Flights, J. Seismol., 6, 62-67, 2002.

Smalley, R. F., Chatelain, J. L., Turcotte, D. L., and Prébot, R.: A fractal approach to the clustering of earthquakes: application to the seismicity of New Hebrides, Bull. Seism. Soc. Am., 77, 4, 1368-1381, 1987.

Sornette, D. and Knopoff, L.: The paradox of the expected time until the next earthquake, Bull. Seism. Soc. Am., 87, 4, 789-798, 1997.

Sotolongo-Costa, O., Antoranz, C., Posadas, A., Vidal, F., and Vázquez, A.: Lévy flights and earthquakes, Geophys. Res. Lett., 27, 13, 1965-1968, 2002.

Takens, F.: Detecting strange attractors in turbulence, in: Lecture Note in Mathematics, edited by: Young, L. S., Springer, Berlin, 1981.

Vidal, F.: Sismotectónica de la región Béticas - Mar de Alborán, $\mathrm{Ph}$. D. Thesis, University of Granada, Spain, 450, 1986.

Wang, J. H. and Kuo, C. H.: On the frequency distribution of interoccurrence times in earthquakes, J. Seismol., 2, 351-358, 1998. 